\title{
Monte Carlo based dosimetry for neutron capture therapy of brain tumors
}

\author{
Lilia Zaidi ${ }^{1}$, Mohamed Belgaid ${ }^{1}$, and Rachid Khelifi ${ }^{2}$ \\ ${ }^{1}$ Faculté de physique USTHB, Laboratoire SNIRM, BP 32 EL-Alia,BEZ Algiers, Algeria. \\ ${ }^{2}$ LPTHIRM, Département de Physique, Université Saad Dahlab, BP 270 Route de Soumaa, Algeria.
}

\begin{abstract}
Boron Neutron Capture Therapy (BNCT) is a biologically targeted, radiation therapy for cancer which combines neutron irradiation with a tumor targeting agent labeled with a boron 10 having a high thermal neutron capture cross section. The tumor area is subjected to the neutron irradiation. After a thermal neutron capture, the excited ${ }^{11} \mathrm{~B}$ nucleus fissions into an alpha particle and lithium recoil nucleus. The high Linear Energy Transfer (LET) emitted particles deposit their energy in a range of about $10 \mu \mathrm{m}$, which is of the same order of cell diameter [1], at the same time other reactions due to neutron activation with body component are produced. In-phantom measurement of physical dose distribution is very important for BNCT planning validation. Determination of total absorbed dose requires complex calculations which were carried out using the Monte Carlo MCNP code [2]
\end{abstract}

\section{Introduction}

During BNCT, the absorbed dose delivered to the normal tissue and tumor, result from a various field irradiation exposure that depends on the spatial, spectral and angular characteristics of the neutron beam as well as on the geometry and composition of the target. The principle absorbed dose components includes: the alpha particles and lithium ions resulting from the boron neutron capture reaction ${ }^{10} \mathrm{~B}(\alpha, \mathrm{n})^{7} \mathrm{Li}$; recoil protons from fast neutron interactions largely due to the ${ }^{1} \mathrm{H}(\mathrm{n}, \mathrm{n}){ }^{1} \mathrm{H}$, and ${ }^{14} \mathrm{~N}(\mathrm{n}, \mathrm{p}){ }^{14} \mathrm{C}$ reactions. All of $(n, \gamma)$ radiative capture reactions particularly the neutron hydrogen capture reaction ${ }^{1} \mathrm{H}(\mathrm{n}, \mathrm{Y})^{2} \mathrm{H}$ were considered [3].

Depending on the position of the tumor, two different neutron beams are commonly used in BNCT: the thermal neutron beam, which limits the treatment to shallow tumors, such as skin melanoma, and the harder epithermal neutron beam as it can penetrate deeper into tissues due to its high energy, it has been used for treating the deepseated tumors. Since we are interested into deep brain tumors such Glioblastoma multiform GBM, the later beam is the adequate one to be used.

In this study, the Radio biological effectiveness (RBE) dose, the depth dose profiles along the central axis of the Snyder's head phantom and in-phantom parameters have been computed for the proposed neutron beam irradiation.

\section{Materials \& Methods}

\subsection{Beam irradiation}

The neutron beam from an accelerator based on the ${ }^{7} \mathrm{Li}(\mathrm{p}, \mathrm{n}){ }^{7} \mathrm{Be}$ reaction [4], with $2.3 \mathrm{MeV}$ proton energy and $10 \mathrm{~mA}$ current, was considered. The primary neutrons from the ${ }^{7} \operatorname{Li}(\mathrm{p}, \mathrm{n})^{7} \mathrm{Be}$ reaction for proton energy of 2.3 $\mathrm{MeV}$ have an average energy of $0.233 \mathrm{MeV}$ which is considered inadequate for treatment with BNCT.

In order to provide an appropriate neutron beam, mainly epithermal neutrons of energy range $(0.5 \mathrm{eV}<\mathrm{E}$ $<20 \mathrm{keV}$ ), a special Beam Shaping Assembly (BSA) must be designed based on the neutron source specifications. A typical BSA includes moderator, reflector, collimator, thermal neutron filter, and gamma filter.

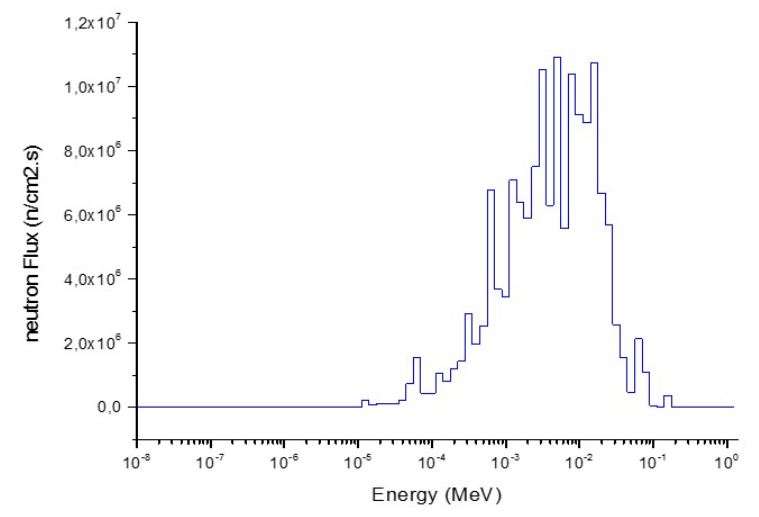

Fig. 1. Neutron beam spectra obtained with the proposed BSA configuration.

In our previous work, detailed studies on materials consisting of the (BSA) was done, where the optimal configuration was chosen as the one can treat tumors in the biggest range of depth and shorter treatment times with best therapeutic ratio. An optimal therapeutic neutron beam presented in Fig.1, centered around $10 \mathrm{keV}$ energy, was obtained. In this study, the beam generated from the BSA is considered as source of irradiation. 


\subsection{Dosimetry}

An ellipsoidal head phantom based on the modified Snyder shown in Fig.2, was considered as a representation of a patient head, the elemental compositions for material of the analytical phantom: scalp, skull and brain have been taken from ICRU 46 [5] of adult head person. The head was positioned at the exit side of the BSA Fig.3.

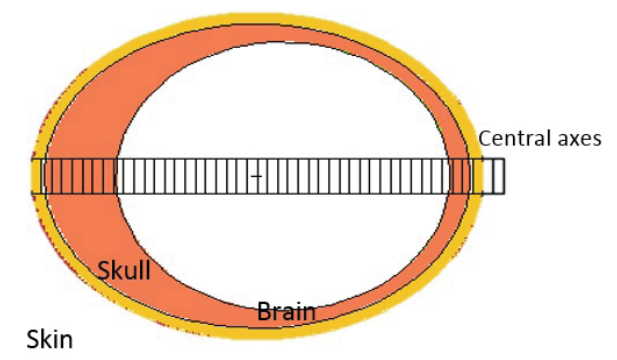

Fig. 2. Snyder head phantom

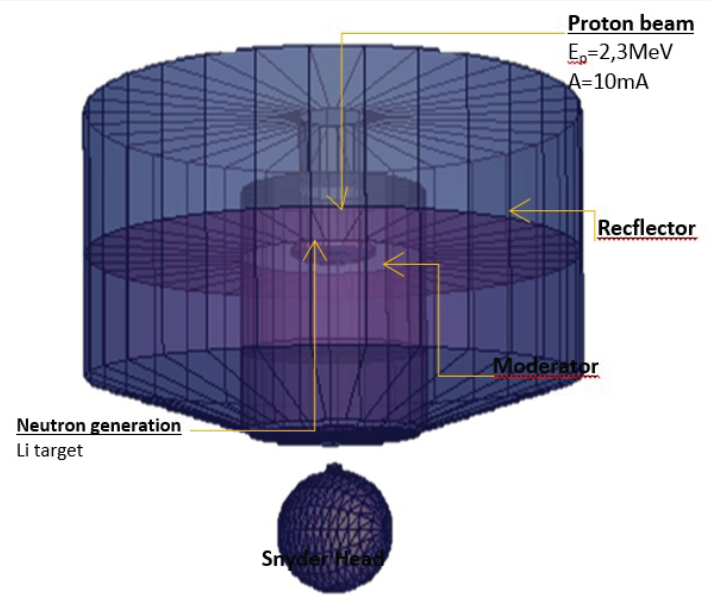

Fig. 3. Schematic view of the BSA and the head phantom configuration simulated for dose calculations.

The absorbed dose from Heavy Charged Particles (HCP) was computed from the neutron flux generated by MCNP for each cell tally in Head phantom. Conversion of particle flux to dose was carried out using the conversion factors for $\mathrm{H},{ }^{16} \mathrm{O},{ }^{14} \mathrm{~N},{ }^{12} \mathrm{C},{ }^{31} \mathrm{P}$ and ${ }^{40} \mathrm{Ca}$ in ICRU 63, based on the ENDF/B-VI nuclear data library [6]. Kerma factors for the elements $\mathrm{Cl}$ and $\mathrm{S}$ were calculated from JENDL3.2 cross section data and Q-values [7].

The gamma ray flux resulting from both the neutron capture gamma rays and the gamma rays from the Litarget and the BSA were converted into absorbed doses using the dose conversion factors based on mass energy absorption coefficient, described by Seltzer [8].

The weighted total dose is defined as the sum of physical dose components multiplied by the weightingfactors of each dose component in a tissue:

$$
D_{w}=w_{g} D_{g}+w_{B} D_{B}+w_{N} D_{N}+w_{f n} D_{f n}
$$

where $D_{w}$ is the weighted total dose, $D_{g}$ is the gamma dose, $D_{B}$ is the absorbed dose due to the boron, $D_{N}$ is the nitrogen dose and $D_{f n}$ is the fast neutron dose. The weighting factors $\mathrm{w}_{\mathrm{N}}$ and $\mathrm{w}_{\mathrm{fn}}$ were taken as $3.2, \mathrm{w}_{\mathrm{g}}$ was considered to be 1.0 and $\mathrm{w}_{\mathrm{B}}$ was considered 1.35 and 3.8 in the normal tissue and tumor, respectively [9].

The ${ }^{10} \mathrm{~B}$ concentrations were assumed to be $52.5 \mathrm{ppm}$ for tumor and $15 \mathrm{ppm}$ for healthy tissue. These four dose components have been computed separately along the center line of the beam through the brain using flux to dose conversion factors using the MCNP F4 tally and DE4/DF4 cards.

\section{Results and discussions}

In-phantom parameters have been calculated in the computational Snyder head phantom model for the particular case of the optimized neutron beam shown in Fig.1. These parameters are: Advantage Depth (AD) (the depth of effective beam penetration at which, the ratio of the dose in the tumour to the dose in the normal tissue, is the maximum); Treatable depth (TD), the depth at which the tumour dose falls below twice the maximum dose to normal tissue, and the treatment time (TT).

Considering that the maximum allowable dose to healthy tissue is $12.5 \mathrm{~Gy}$, TT can be estimated. On the other hand, In BNCT, treatment time can extend up to one hour. Results in Table 1 shows the estimated parameters for the neutron beam generated by the optimized BSA, and the treatment capabilities.

\begin{tabular}{|c|c|}
\hline Parameters & Values \\
\hline Total dose (RBE.Gy/min) & 0,519 \\
\hline Max Tumor dose (RBE. Gy/min) & $4,31 \mathrm{E}-01$ \\
\hline Mean Brain dose (RBE.Gy/min) & $4,140486 \mathrm{E}-2$ \\
\hline Skin dose (RBE.Gy/min) & $3,11 \mathrm{E}-02$ \\
\hline Dose ratio or therapeutic gain & 4,92 \\
\hline Treatment time TT (h) & 1 \\
\hline Treatable depth TD (cm) & 7,1 \\
\hline $\begin{array}{c}\text { Maximum healthy brain punctual } \\
\text { dose 11 RBEGy }\end{array}$ & 4,8 \\
\hline
\end{tabular}

The Fig. 4. shows the depth profile of the dose components rate for healthy tissue and tumour.

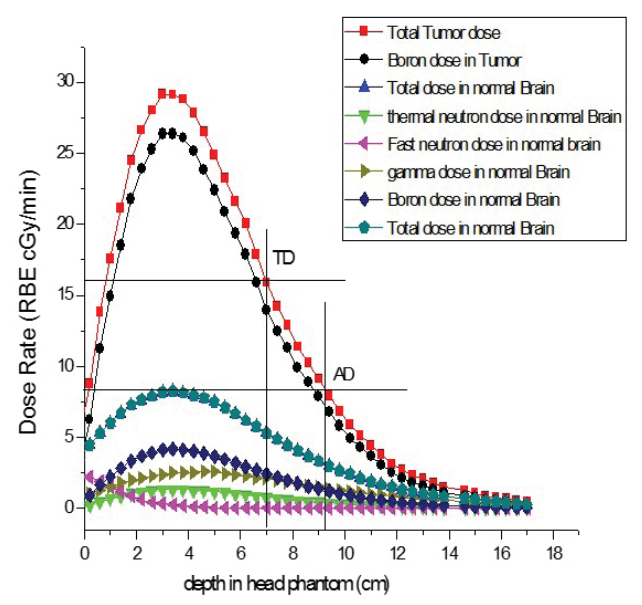

Fig. 4. Distribution of various dose components in the head 
In the brain the boron component due to nonspecific boron deposition in healthy tissue dominates. The gammas dose is the second component contribution, mainly due to gammas produced inside the phantom. These two components are the limiting factors in healthy brain while at the tumour tissue the dominant dose component is due to Boron dose. The dose contribution from hydrogen recoil due to fast neutrons reactions on hydrogen and nitrogen still small.

\section{Conclusions}

In this study, by means of numerical simulations, the RBE dose, depth dose profiles have been computed and the beam quality was also characterized with the help of the dose estimation in the Snyder's head phantom.

The obtained results in this work, for the considered neutron beam source, show good treatment possibilities with short irradiation times. The beam penetration is good: maximum tumor dose at $3.4 \mathrm{~cm}$ depth; and an advantage depth of $9.2 \mathrm{~cm}$. with the maximized dose ratio 4.92 of tumor to normal tissue. Then the designed beam encompass suitable quality and intensity, it can deliver more than 25.8 RBE Gy to a tumor in one hour for only $10 \mathrm{~mA}$ proton current.

\section{References}

1. IAEA, International Atomic Energy Agency, IAEATECDOC-1223, ( 2001).

2. J.F. Briesmeister, RSICC, Oak Ridge National Laboratory, LANL manual, LA-12625-M, Los Alamos, (1997).

3. J. T. Goorley, W. S. Kiger, and R. G. Zamenhof, Med. Phys., 29, 145-156, (2002).

4. C. L. Lee and X. Zhou, 152, 1-11, (1999).

5. ICRU 46, International Commission on Radiation Units and Measurements Tech. rep. (1992).

6. Brookhaven National Laboratory, "ENDF/B-VI Summary Documentation," BNL-NCS-17541, (1991).

7. T. Nakagawa, K. Shibata, S. Chiba, T. Fukahori, Y. Nakajima, Y. Kikuchi, T. Kawano, Y. Kanda, T. Ohsawa, H. Matsunobu, M. Kawai, A. Zukeran, T. Watanabe, S. Igarasi, K. Kosako, and T. Asami, "Japanese Evaluated Nuclear Data Library Version 3 Revision 2: JENDL-3.2," J. Nucl. Sci. Technol., 32, 1259 (1995).

8. J.H. Hubbell, S.M. Seltzer. NISTIR 5632, National Institute of Standards and Technology, (1995).

9. D.T. Ingersoll, et al., in Advances in Neutron Capture Therapy, Vol. I, edited by B. Larsson, J. Crawford, and R. Weinreich, Elsevier, Amsterdam (1997), pp. 95-99 